\title{
Functions of the Triceps Brachii in Humans: A Review
}

\author{
Dennis Landin ${ }^{\mathrm{a}, \mathrm{b}}$, Melissa Thompson ${ }^{\mathrm{a}}$, Meghan Jackson ${ }^{\mathrm{a}}$
}

\begin{abstract}
The triceps brachii (TB) is the only muscle that lies along the posterior humerus, and its role in elbow extension has been the topic of investigation for many decades. The TB research has also included subjects such as strength training, effects of aging, training adaptions, etc. The purpose of this paper was to review some of the more unique investigations regarding the functions of the human TB. Specifically, this paper discusses articles that have explored the actions of the TB when manipulating the angles of the joints it crosses, the elbow and shoulder.
\end{abstract}

Keywords: Triceps Brachii; Elbow extension; Shoulder extension

\section{Introduction}

The triceps brachii (TB) is the only muscle that lies along the posterior humerus. It is a three-headed, fusiform muscle that, at the shoulder, works in a third-class lever since the force is applied between the joint axis and the load [1]. The TB is a bi-articular muscle for it also crosses the elbow, where it again operates in a third-class lever system. Such lever systems are specialized for range and speed of motion, and the extremities of the human body contain mostly third-class levers. Its proximal attachments are the infraglenoid tubercle, the lateral humerus, and the posterior/medial humerus for the long, lateral and medial heads, respectively. The medial head of the triceps has a tendon that lies deep to, and is initially separate from, the tendon shared by the long and lateral heads [2]. The tendons of all three heads insert on the olecranon process of the ulna. With the long head arising from the infraglenoid tubercle, the TB affects motion at both joints by acting as an extensor at each [1, 3-6]. Since most muscle action descriptions were developed without the benefit of today's technology, the extent to which the TB extends the shoulder has received less attention. With equipment such as isokinetic dynamometers now available, it is possible to collect precise information on a muscle's actions while controlling multiple joints.

Manuscript submitted December 27, 2017, accepted January 29, 2018

aSchool of Kinesiology, Louisiana State University, Baton Rouge, LA, USA ${ }^{b}$ Corresponding Author: Dennis Landin, School of Kinesiology, Louisiana State University, Baton Rouge, LA 70810, USA. Email: dlandin@1su.edu

doi: https://doi.org/10.14740/jocmr3340w

\section{General Functions}

The elbow extension work of the TB has been known and studied extensively, for more than a century [3], and it clearly is a powerful extender of the forearm. The TB's elbow extensor work is enhanced, to a small degree, by the mon-articular anconeus [4-6]. Salmons [1] indicates that the medial head is involved in all forms of elbow extension, while the long and lateral heads become active only when the elbow is extended against resistance. Additional details on the TB's actions were provided by Madsen et al [2] who showed that the three heads of the TB do not necessarily work as a single unit throughout the extension movement. Through the examination of the upper extremities of eight cadavers, Madsen and his colleagues [2] found that the medial head was attached to the olecranon via a deeper and separate tendon. They concluded that the medial head becomes fully involved in extension only when the elbow is flexed beyond $90^{\circ}$. Despite this subtle variation, elbow extension is still its primary function. In regards to the shoulder, its role is less well defined. Since the proximal attachment of the long head crosses the shoulder, albeit minimally, anatomists have traditionally included the TB among the muscles producing movement at this joint $[1,4,5]$. It works in shoulder (humeral) extension, although there are several other muscles (e.g., latissimus dorsi, posterior fibers of the deltoid, and teres major and minor) that contribute to this action. It also has a part in shoulder (humeral) adduction, although other muscles again are more critical (e.g., pectoralis major, latissimus dorsi). Moore and Dalley [7] point out that the TB, due to its bi-articular nature, also plays a role in stabilizing the abducted glenohumeral joint by resisting inferior displacement of the humeral head. The TB role at the shoulder is considered so minor that clinical examination of the shoulder often excludes the TB, or stresses evaluation as a shoulder extensor only when the elbow is flexed [8].

\section{Joint Angles Influence Function}

Investigations into the function of the TB in elbow extension, and as a focus of attention in various muscle tissue topics, have been conducted for years [3, 9-12]. This review sought out TB articles that have explored how changes in elbow and/or shoulder angles affect the TB functions.

Murray et al [13] investigated the isometric capacity of the muscles which cross the elbow in 10 unembalmed cadaver extremities. These investigators measured muscle fascicle length, sarcomere length, pennation angle, mass, and tendon displacement during elbow flexion. From these data, optimal 


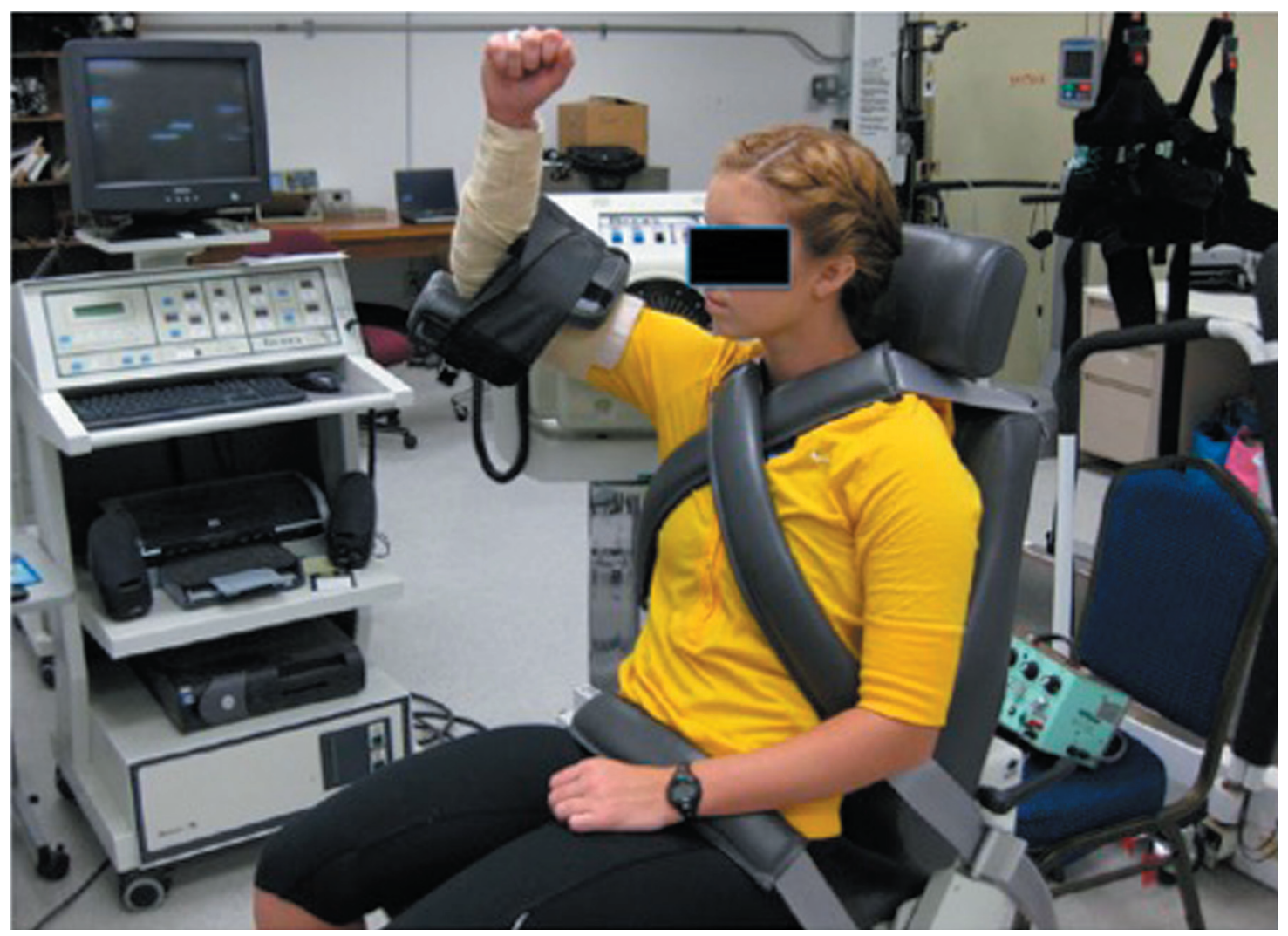

Figure 1. Subject position during testing [16].

fascicle length, physiological cross-sectional area (PCSA), moment arm, and operating range on the force-length curve were estimated. One of the muscles studied was the TB, and some of that information is useful in this review. The TB (combined heads), in these specimens, had the greatest PCSA of the muscles studied, but had a relatively short range of operation over the force-length curve due to a short moment arm (2.3 $\mathrm{cm})$. The large PCSA of the TB, though, gave this muscle the largest moment-generating potential. Particularly pertinent to this review is the finding that the long head of the TB maintained a relatively constant force-generating capacity across a wide range of elbow angles.

In a unique follow-up paper, Murray et al [14] explored the effect of bone width on peak moment arms of selected muscles. The authors pointed out that peak moment arms should correspond with the shortest distance (the investigators labeled this " $\mathrm{D}_{\mathrm{s}}$ ") between the axis of rotation, and the muscle's proximal and distal attachments. However, they argued that since muscle attachments near the elbow wrap around the bones, then the anterior-position dimensions of the bone would influence the moment arm. Using the extremities from 10 unembalmed cadavers, Murray et al reported that peak moment arms did correlate strongly with $\mathrm{D}_{\mathrm{s}}$ for muscles such as the brachioradialis, biceps brachii, and extensor carpi radialis longus. But for the TB, and a few others, this correlation was weak. In fact, for the TB, the anterior-posterior dimensions of the ulna accounted for over $60 \%$ of the variation in peak moment arms across the specimens.
Similar to the work of Murray et al [13, 14], Doheny et al [15] explored how the elbow angle affected the relationship between muscle force and muscle activations values recorded with electromyography (EMG). The EMG data were acquired from the brachioradialis, biceps brachii, and TB muscles. Using eight elbow angles between $10^{\circ}$ and $120^{\circ}$, where a fully extended elbow was considered $0^{\circ}$, the subjects performed maximal voluntary contractions (MVCs) which were held for $2 \mathrm{~s}$. EMG recordings were collected during each trial. Dohney and his colleagues [14] reported that the elbow angle had a significant effect on MVC force but not on MVC EMG amplitude. More specifically, these results showed that the forces generated by the MVC during flexion and extension were altered as the elbow angle changed. For both directions, the MVC was lowest near the $0^{\circ}$ position and then increased in a linear fashion reaching its peak around $80^{\circ}$. Elbow positions greater than $80^{\circ}$ were marked by a progressive decline.

The work of Murray et al [13, 14] offers important information on how muscle structure and the configuration of the attachments will influence its power and functionality, while the work of Doheny et al [15] showed that changing the angle of the elbow altered flexion and extension forces. However, since bi-articular muscles are affected by two joints, a more complete understanding of their functions requires that the combined effects of the angles of both joints be considered, and a search of the literature revealed very little work with the TB when both joints were manipulated.

In our TB paper [16], we explored the shoulder extension 
Table 1. Shoulder Extension Maximum Moment (Nm) [16]

\begin{tabular}{|c|c|c|c|c|c|}
\hline \multirow{2}{*}{ Elbow angle $\left(^{\circ}\right)$} & \multicolumn{5}{|c|}{ Shoulder angle $\left({ }^{\circ}\right)$} \\
\hline & $\mathbf{0}$ & 40 & 80 & 120 & Mean \\
\hline 0 & $0.75(0.18)$ & $7.41(0.53)$ & $12.58(0.85)$ & $12.94(0.90)$ & 8.42 \\
\hline 60 & $2.12(0.35)$ & $9.58(0.27)$ & $14.25(0.42)$ & $12.47(0.96)$ & 9.6 \\
\hline 90 & $2.5(0.27)$ & $9.25(0.20)$ & $12.94(0.29)$ & $10.72(0.86)$ & 8.85 \\
\hline Mean & 2.16 & 8.71 & 13.42 & 12.26 & \\
\hline
\end{tabular}

Mean: mean difference $>4.03$ are significant.

(SX) capabilities of the TB in 16 different combinations of elbow and shoulder angles. Four elbow angles $0^{\circ}$ (the anatomical position), $30^{\circ}, 60^{\circ}$, and $90^{\circ}$ were crossed with four shoulder angles $0^{\circ}$ (anatomical position), $40^{\circ}, 80^{\circ}$, and $120^{\circ}$. This produced 16 combinations, all positioned within the scapular plane. At each position, the TB of the subject's right arms was stimulated three times with surface electrodes. Figure 1, from our 2011 paper [16], shows the experimental setup for the $90^{\circ} / 120^{\circ}$ elbow/shoulder (ES) position. In our 2011 paper, we reported on three dependent measures. The passive moment was the torque produced by the resting TB and other passive tissue as the length changed across the joint combinations. Second dependent measure was the stimulated moment, which the torque produced during the electrical stimulation minus the passive tissue contribution. The third dependent measure, and most germane to this review, was the maximum moment (MM) which represented the combined passive and stimulated moments. Table 1, from our 2011 paper [16], contains the maximum shoulder extension moments, expressed in Newton/meters $(\mathrm{Nm})$, produced by the TB at each combination of joint angles. Analysis of the data revealed that the shoulder angle was the only significant factor in MM levels, and that the MM increased in a linear fashion as the shoulder moved from $0^{\circ}$ to $80^{\circ}$. Elevating the shoulder beyond $80^{\circ}$ resulted in a small decrease in the MM. While the elbow was not a significant factor, it was the $60^{\circ}$ position that proved most favorable. Doheny et al [15] did not investigate the effect of the shoulder angle on the TB MVC in elbow flexion using only various elbow angles. With the shoulder held in the $0^{\circ}$ (anatomical) position, Doheny et al reported that the $60^{\circ}$ elbow angle was most favorable for TB to extend the forearm. In our paper [16], we found that the 60/80 ES position produced the highest MM for SX, so the Doheny et al's elbow position and our 60/80 ES were similar even though the different joint movements were measured. An interesting experiment would be to combine the Doheny et al's procedures with ours by having the subjects perform MVCs at the 16 elbow/ shoulder angles we used.

Further results from our 2011 [16] paper showed that, compared to the shoulder, the elbow had little effect on the SX moment. In the 0/0 ES position, there was nearly no SX moment recorded. Even as the elbow moved toward $90^{\circ}$, there was very little SX torque if the shoulder remained at $0^{\circ}$. Once the shoulder angle moved away from $0^{\circ}$, then the SX moment began to rise. We argued that these results suggest that the elbow does not substantially alter the length of the TB even as it approaches $90^{\circ}$. This may be explained by the TB's insertion point on the ulna, which is on the most proximal border of the olecranon [1] and does not appear to displace much during elbow flexion. As discussed in a previous paragraph, Murray et al [14] reported that the anterior-posterior dimensions of the ulna, at the insertion of the TB, accounted for over $60 \%$ of the variation in peak moment arms for elbow extension across cadaveric specimens. Reconciling this result with ours [16] requires being mindful that Murray et al did not manipulate the angle of the shoulder but focused only on the elbow. We found the shoulder angle to be the critical variable in TB SX moments; consequently, the variations in ulnar anterior-posterior dimensions apparently become a factor only in elbow extension moments and do not have much importance in the SX function of the TB. It can be argued that, since many actions of the upper extremities are multi-joint motions, considering the elbow angle in isolation may confound the study of TB peak moments.

\section{Conclusion}

This review discussed the actions of the TB and some of the influencing factors. The vast majority of the TB research has considered its actions only at the elbow. Our study [16] is the only one in the literature which explored how various combinations of shoulder and elbow angles affect the TB's shoulder extension actions. Two intriguing findings emerged. First, the anterior/posterior dimensions of the ulna, at the point where the TB inserts, can influence its elbow extension torque. Murray et al [14] reported that these ulnar dimensions accounted for over $60 \%$ of the variation in peak moment arms in cadaveric specimens. Second, in regards to the TB's role in SX, the degree to which the shoulder is elevated is the most important factor in determining the TB SX moments. Elevating the shoulder from $0^{\circ}$ to $40^{\circ}$ produces a dramatic change in the SX torque of the TB, and this continues to increase up to the $80^{\circ}$ position. Elevating the shoulder beyond $80^{\circ}$ can result in a small decrease in the SX MM.

\section{References}

1. Salmons S. Muscle. In: Williams PL, Bannister LH, Berry MM, Collins P, Dyson M, Dussek JE, Ferguson MWJ, editors. Gray's anatomy. New York: Churchill, 1995:737- 
900 .

2. Madsen M, Marx RG, Millett PJ, Rodeo SA, Sperling JW, Warren RF. Surgical anatomy of the triceps brachii tendon: anatomical study and clinical correlation. Am J Sports Med. 2006;34(11):1839-1843.

3. Pickering T, Howden R. Muscle. Grays' anatomy. 15th ed. Facsimile; 1901. Reproduced by Barnes and Nobles (1995).

4. Tortora GJ. Principles of human anatomy. Hoboken, NJ: Wiley and Sons, 2005.

5. Van De Graaff KM. Human anatomy. In: Dubuque IA, editor. McGraw-Hill, 2002.

6. Williams PL, Warwick R, Dyson M, Bannister LH, editors. Gray's anatomy. 37th ed. 1989.

7. Moore KL, Dalley AF. Clinically oriented anatomy. Philadelphia: Lippincott Williams and Wilkins; 1999.

8. Starkey C, Ryan J. Evaluation of orthopedic and athletic injuries. Philadelphia: F.A. Davis Company; 2002.

9. Kawakami Y, Abe T, Kuno SY, Fukunaga T. Training-induced changes in muscle architecture and specific tension. Eur J Appl Physiol Occup Physiol. 1995;72(1-2):37-43.

10. Ma SP, Zahalak GI. The mechanical response of the ac- tive human triceps brachii muscle to very rapid stretch and shortening. J Biomech. 1985;18(8):585-598.

11. Rice CL, Cunningham DA, Paterson DH, Dickinson JR. Strength training alters contractile properties of the triceps brachii in men aged 65-78 years. Eur J Appl Physiol Occup Physiol. 1993;66(3):275-280.

12. Winters JM, Kleweno DG. Effect of initial upper-limb alignment on muscle contributions to isometric strength curves. J Biomech. 1993;26(2):143-153.

13. Murray WM, Buchanan TS, Delp SL. The isometric functional capacity of muscles that cross the elbow. J Biomech. 2000;33(8):943-952.

14. Murray WM, Buchanan TS, Delp SL. Scaling of peak moment arms of elbow muscles with upper extremity bone dimensions. J Biomech. 2002;35(1):19-26.

15. Doheny EP, Lowery MM, Fitzpatrick DP, O’Malley MJ. Effect of elbow joint angle on force-EMG relationships in human elbow flexor and extensor muscles. J Electromyogr Kinesiol. 2008;18(5):760-770.

16. Landin D, Thompson M. The shoulder extension function of the triceps brachii. J Electromyogr Kinesiol. 2011;21(1):161-165. 